Annuaire suisse de politique de développement

23-1 | 2004

Faits et statistiques 2004

\title{
7. Relations financières internationales
}

\section{Bruno Gurtner}

\section{OpenEdition}

Journals

Édition électronique

URL : http://journals.openedition.org/aspd/475

DOI : 10.4000/aspd.475

ISSN : 1663-9669

\section{Éditeur}

Institut de hautes études internationales et du développement

\section{Édition imprimée}

Date de publication : 1 avril 2004

Pagination : 105-130

ISSN : 1660-5934

\section{Référence électronique}

Bruno Gurtner, «7. Relations financières internationales », Annuaire suisse de politique de

développement [En ligne], 23-1 | 2004, mis en ligne le 12 mars 2010, consulté le 08 septembre 2020 URL : http://journals.openedition.org/aspd/475 ; DOI : https://doi.org/10.4000/aspd.475 


\section{Relations financières internationales*}

L

ES FLUX de capitaux publics et privés à destination des pays en développement et en transition ont continué de baisser en 2002, tendance qui a toutefois pu être stabilisée en 2003. Ce recul a été particulièrement marqué pour les investissements directs étrangers. Bien que leur endettement extérieur n'ait plus guère augmenté dans l'ensemble, de nombreux pays en développement restent confrontés à de graves problèmes financiers. Quelques-uns voient leur dette publique gonfler dans des proportions très inquiétantes. Le volume des crédits octroyés par les institutions de Bretton Woods est resté considérable.

\subsection{Flux financiers vers les pays en développement et en transition}

Les pays en développement financent neuf dixièmes des investissements effectués sur leur territoire à partir de ressources intérieures. Ils restent cependant tributaires des afflux de capitaux étrangers. Mais ces afflux ont diminué en 2002 comme les années précédentes, aussi bien pour les capitaux publics que les capitaux privés. Le volume des crédits continue de baisser, et les transferts de personnes migrantes sont devenus la deuxième source de financement.

Les flux de capitaux investis dans les pays en développement et en transition ont encore diminué par rapport à l'année précédente, tombant ainsi, selon les données de la Banque mondiale dans Global Development Finance $(G D F)^{1}$, à 192 milliards de dollars en 2002 (voir tableau 28). Ce montant correspond globalement à 3,2\% du PNB nominal des pays en développement.

Le Fonds monétaire international (FMI) et la Banque des règlements internationaux (BRI) indiquent des tendances analogues quant aux flux de capitaux ${ }^{2}$. L'Institute of International Finance (IIF), centre d'études des grandes banques commerciales, présente de son côté des chiffres sur les flux financiers vers les marchés émergents ${ }^{3}$.

Ce recul est surtout le fait des fournisseurs de capitaux privés, mais concerne aussi les fonds publics. Les financements publics à taux préférentiels ont toutefois augmenté pour la première fois depuis un certain temps. Selon des estimations provisoires du FMI et de la Banque mondiale, cette baisse des flux

* Par Bruno Gurtner, économiste, responsable des relations financières internationales à la Communauté de travail Swissaid/Action de carême/Pain pour le prochain/Helvetas/Caritas/EPER.

1 The World Bank (ci-après WB), Global Development Finance: Striving for Stability in Development Finance, vol. 1: Analysis and «Statistical Appendix», Washington, 2003, <www.worldbank.org/ prospects/gdf2003/tocvol1.htm>, et IMF/WB, Development Committee, Recent Trends in Financial Flows to Developing Countries, Washington, 27 August 2003, <www.worldbank.org/ambc >.

2 Voir surtout: IMF, World Economic Outlook: Growth and Institutions, April 2003, <www.imf. org/external/pubs/ft/weo/2003/01> ; IMF, World Economic Outlook: Public Debt in Emerging Markets, September 2003, <www.imf.org/external/pubs/ft/weo/2003/02> ; IMF, Global Financial Stability Report, divers numéros, <www.imf.org/external/pubs/ft/gfsr/about.htm> ; BRI, diverses publications (rapport annuel, rapports trimestriels, etc.), <www.bis.org $>$.

3 IIF, Capital Flows to Emerging Markets Economies, diverses publications, <www.iif.com >. 
pourrait ralentir en ce qui concerne les capitaux privés au cours de 2003 et même céder la place à une légère reprise en 2004.

Cela s'explique d'un côté par la situation difficile que vit l'économie mondiale. Alors que l'on pronostiquait à maintes reprises un nouvel essor, il a fallu chaque fois réviser à la baisse ces prévisions de croissance - caractérisées de toute manière par d'énormes disparités régionales.

Par ailleurs, les pays en développement ont eu énormément de peine à s'adapter aux transformations structurelles à long terme qu'ont subies les flux financiers. Au cours de ces dernières années, les financements par capitaux de tiers (crédits bancaires, obligations) ont de plus en plus cédé la place au financement de capitaux propres. Les capitaux étrangers continuent de fuir les pays en développement.

Tableau 28: Flux de capitaux à destination des pays en développement, 1997-2003 (en milliards de dollars aux taux actuels)

\begin{tabular}{lrrrrrrr}
\hline & $\mathbf{1 9 9 7}$ & $\mathbf{1 9 9 8}$ & $\mathbf{1 9 9 9}$ & $\mathbf{2 0 0 0}$ & $\mathbf{2 0 0 1}$ & $\mathbf{2 0 0 2}^{\mathbf{a}}$ & $\mathbf{2 0 0 3}^{\mathrm{b}}$ \\
\hline $\begin{array}{l}\text { Financement public } \\
\text { du développement }\end{array}$ & 36.7 & $\mathbf{4 8 . 2}$ & $\mathbf{4 5 . 0}$ & $\mathbf{3 4 . 0}$ & 38.1 & $\mathbf{3 4 . 5}$ & 32.0 \\
\hline à conditions de faveur & 34.1 & 37.7 & 41.2 & 36.5 & 37.8 & 43.0 & \\
\hline \multicolumn{1}{c}{ dons } & 26.7 & 28.2 & 29.4 & 29.6 & 29.5 & 32.9 & \\
\hline \multicolumn{1}{c}{ crédits à taux préférentiels } & 7.4 & 9.5 & 11.8 & 6.9 & 8.3 & 10.2 & \\
\hline crédits non préférentiels & 2.2 & 10.5 & 3.8 & -2.8 & 0.3 & -8.5 & \\
\hline Flux de capitaux privés & 280.0 & 269.4 & 216.2 & 201.1 & 169.1 & 143.3 & 163.0 \\
\hline crédits bancaires & 43.1 & 51.4 & -5.9 & 2.6 & -11.8 & -16.0 & \\
\hline prêts obligataires & 38.4 & 39.7 & 29.6 & 17.4 & 10.1 & 18.6 & \\
\hline autres & 2.5 & -3.6 & -1.8 & -5.5 & -7.0 & -5.5. & \\
\hline investissements de portefeuille & 26.7 & 7.4 & 15.0 & 26.0 & 6.0 & 9.4 & 13.0 \\
\hline investissements directs étrangers & 169.3 & 174.5 & 179.3 & 160.6 & 171.7 & 143.0 & 145.0 \\
\hline Total & $\mathbf{3 1 6 . 3}$ & $\mathbf{3 1 7 . 7}$ & $\mathbf{2 6 1 . 2}$ & $\mathbf{2 3 5 . 1}$ & $\mathbf{2 0 7 . 2}$ & $\mathbf{1 9 2 . 3}$ & $\mathbf{1 9 5 . 0}$ \\
\hline
\end{tabular}

Sources: WB, Global Development Finance 2003, vol. 1, tableaux 1.1 et A.22; IMF/WB, Development Committee, Recent Trends in Financial Flows to Developing Countries, Washington, 27 August 27 2003, <www.worldbank.org/ambc>.

a Estimation provisoire.

b Prévision.

\section{$\square$ Flux financiers privés}

Selon la Banque mondiale, les flux de capitaux privés à destination des pays en développement sont tombés à 143 milliards de dollars en 2002. La Banque pronostique cependant une légère reprise en 2003. Les différentes formes de flux privés ont contribué de façon variable à ce résultat global.

Le remplacement du financement par capitaux de tiers (crédits bancaires, obligations) par un financement par capitaux propres (investissements directs étrangers, investissements de portefeuille) se confirme à moyen terme; cette évolution s'explique par une prudence accrue des investisseurs. C'est pour les crédits bancaires que le recul est le plus marqué: les banques ont accordé moins de crédits qu'elles n'ont obtenu de remboursements. Les sorties de capitaux des pays en développement ont dépassé les entrées à hauteur de 16 milliards de dollars en 2002. Et le niveau des prêts bancaires est resté faible durant le premier semestre 2003.

Plutôt que d'accorder elles-mêmes des crédits, les banques privées préfèrent offrir leurs services aux créanciers et aux débiteurs de prêts obligataires (bonds). Mais les dernières crises financières ont également conduit les investis- 
seurs privés à éviter des risques et à privilégier les obligations solides, ce qui explique en partie les fortes fluctuations subies par celles-ci. Bien des pays en développement ont été à même d'émettre plus facilement des obligations en 2002, tendance qui s'est maintenue durant le premier semestre 2003. L'Amérique et l'Europe de l'Est en ont bien profité, tandis que les pays asiatiques ont moins lancé de prêts obligataires. En particulier, le Brésil a ainsi pu obtenir de nouveaux fonds après plus d'une année d'absence sur le marché des capitaux. Cependant, les faibles niveaux de solvabilité se voient sanctionnés par de fortes majorations des intérêts.

Les investissements directs étrangers ont eux aussi marqué une baisse et totalisent 143 milliards de dollars en 2002 (172 milliards en 2001). Les premiers chiffres pour l'année 2003 témoignent d'une situation qui continue de stagner. Elle est due à la faiblesse du marché international des titres et au nombre décroissant de privatisations et de fusions. Ce recul a été particulièrement marqué en Amérique latine, tandis que la Chine, l'Inde et l'Europe de l'Est voyaient ces investissements augmenter ${ }^{4}$.

Les investissements de portefeuille ont en revanche passé de 6 à 9 milliards de dollars avec une tendance à la progression qui se maintient en 2003, mais ils restent nettement au-dessous des sommets atteints au début des années 1990. L'Asie orientale s'est taillé la part du lion, tandis que les flux à destination de l'Amérique latine ont régressé.

\section{$\square$ Financement public du développement}

Atteignant 34,5 milliards de dollars en 2002, le financement public du développement a lui aussi baissé par rapport à l'année précédente (49 milliards). Cette évolution est due exclusivement à la nette diminution des crédits à taux d'intérêt non préférentiels accordés par les institutions multilatérales, combinée aux remboursements considérables de crédits de même type à des donateurs bilatéraux ${ }^{5}$. Les crédits à taux préférentiels de donateurs multilatéraux aussi bien que bilatéraux, et surtout l'aide publique au développement sous forme de dons, ont par contre marqué une légère augmentation pour la première fois depuis longtemps. La Banque mondiale attribue cela aux promesses faites lors de la Conférence de Monterrey en 2002 concernant le financement du développement. Elle a par ailleurs fait observer que cette aide ne profite pas intégralement aux pays en développement: les salaires des experts et consultants ainsi que les frais administratifs restent dans les pays industrialisés.

L'OCDE confirme que l'aide publique au développement a augmenté. Les pays du CAD ont versé à ce titre, en 2002, un total de 57 milliards de dollars, soit $4,9 \%$ de plus que l'année précédente ${ }^{6}$. Cela correspondait à $0,22 \%$ de leur PNB $(0,22 \%$ en 2001).

Annuaire 2004, $n^{\circ}$ 1, partie 2.1, «Volume de l'APD des pays du CAD et de la Suisse».

\footnotetext{
Voir plus bas la partie 7.2 sur les investissements internationaux. WB, GDF 2003, tableau 6.1, p. 126.

OCDE, CAD, Les pays de l'OCDE membres du CAD amorcent un redressement de leur APD, avec une progression de $5 \%$ en 2002, communiqué de presse, 21 avril 2003, <www.oecd.org>. Concernant la différence entre les chiffres de la Banque mondiale et ceux de l'OCDE, voir Annuaire 2003, $\mathrm{n}^{\circ} 1$, p. 98, n. 3 .
} 
Douze des 22 pays membres du CAD ont accru leur aide, dont les EtatsUnis - lesquels continuent cependant de s'en tenir au niveau médiocre de $0,12 \%$ de leur PNB. De nombreux pays ont promis d'augmenter - substantiellement pour certains - leur aide ces prochaines années. L'OCDE constate pour la Suisse une légère progression en chiffres absolus de 25 millions de dollars qui porte le total à 933 millions. Mais l'aide helvétique a proportionnellement régressé de 0,34 à 0,32\% du PNB, ce que l'OCDE attribue (comme pour la Grande-Bretagne) à un retard dans les versements à effectuer pour reconstituer le capital de l'AID, filiale de la Banque mondiale.

\section{$\square$ Transferts des migrants}

La Banque mondiale indique que les versements effectués dans leur pays d'origine par les personnes migrantes constituent la deuxième en importance des sources de financement externe, derrière les investissements directs étrangers ${ }^{7}$. Ces transferts ont atteint 80 milliards de dollars en 2002. Ils ont continuellement augmenté ces dernières années, avec une régularité remarquable en dépit des fluctuations conjoncturelles subies par les pays où séjournent les migrants. Les principaux pays destinataires sont l'Inde et le Mexique (10 milliards de dollars chacun), suivis par les Philippines (6 milliards) et le Maroc (3 milliards). Les transferts de migrants représentaient $37 \%$ du PNB des îles Tonga, 26,5\% du Lesotho et $22,8 \%$ de la Jordanie. Mais cette proportion n'est de loin pas aussi importante dans la plupart des pays. Les transferts de fonds réalisés par des travailleurs étrangers ont contribué à hauteur de 1,3\% au PNB des pays en développement dans leur ensemble. Cette proportion atteint une moyenne de 1,9\% pour les pays les plus pauvres.

L'argent sorti des Etats-Unis par les travailleurs étrangers a totalisé 28 milliards de dollars. Bien qu'il soit difficile de chiffrer la répartition de ces transferts de fonds sur les différents pays destinataires, la Banque mondiale estime la part des pays en développement à 18 milliards de dollars pour l'année 2001. Le deuxième en importance des pays d'origine de ces transferts est l'Arabie saoudite (15 milliards), suivie, toujours selon la Banque mondiale, par l'Allemagne, la Belgique et la Suisse avec chacune 8 milliards de dollars. La Banque mondiale n'indique pas combien d'argent passe ainsi de la Suisse vers des pays en développement et en transition.

\section{$\square$ Exportations de capitaux des pays en développement}

Comme de nombreux pays en développement ont considérablement augmenté leurs réserves de devises, le Sud - si l'on ajoute les remboursements de dettes - présente un solde exportateur de capitaux vers le Nord: c'est ce qu'explique brièvement la Banque mondiale dans l'introduction de son dernier rapport $G D F$, sans revenir sur la question abordée l'année précédente concernant les sorties de capitaux qui échappent aux statistiques. Une étude privée montre cependant, en se référant aux statistiques relatives à la balance des paiements, que les flux de capitaux sont plus importants du Sud vers le Nord qu'en sens inverse. Etonnamment, c'est également le cas pour des pays à forte croissance

7 WB, GDF 2003, chap. 7, pp. 157 sqq., où l'on trouvera d'autres références bibliographiques ainsi qu'un commentaire sur les problèmes posés par les sources de données. 
tels que la Chine, la Corée du Sud ou Taïwan ${ }^{8}$. De même, la CNUCED signale que le bilan des transferts financiers (transferts de capitaux englobant service de la dette et intérêts ainsi que rapatriement de bénéfices) présentait un solde négatif de 192,5 milliards de dollars ${ }^{9}$.

\section{$\square$ Facilité financière internationale}

Gordon Brown, ministre britannique des Finances, a proposé en novembre 2002 de créer une nouvelle International Finance Facility (IFF) que les pays donateurs s'engageraient à alimenter par des versements réguliers. Ces garanties permettraient à l'institution de faire des emprunts à taux d'intérêt avantageux sur le marché international des capitaux. Et les fonds ainsi obtenus pourraient être mis à la disposition des pays en développement par les canaux bilatéraux et multilatéraux existants, à des conditions plus avantageuses que si les pays emprunteurs devaient s'approvisionner eux-mêmes sur le marché des capitaux. Il devrait être ainsi possible de réunir des fonds toujours plus importants jusqu'en 2015. Les dettes accumulées de cette manière seraient ensuite remboursées entre 2015 et $2030^{10}$.

Cette proposition a eu jusqu'à présent relativement peu d'échos sur la scène internationale. Il est seulement question d'étudier, lors de la session de printemps des institutions de Bretton Woods, si une IFF pourrait faire figure de variante par rapport aux mécanismes de financement actuels. La Suisse y a ellemême réagi négativement, selon des déclarations orales du Département fédéral des finances. Ce plan aurait pour effet d'hypothéquer de futures prestations de développement. A l'accroissement jusqu'en 2015 des flux financiers vers les pays les plus pauvres ferait suite une diminution symétrique après cette échéance. La Suisse préférerait entreprendre des efforts supplémentaires en passant par les structures multilatérales existantes.

\subsection{Investissements internationaux}

L'afflux des investissements directs étrangers a encore ralenti, et les pays en développement en ont également souffert. La part de ce type d'investissements reste faible dans les pays les plus pauvres. De même, les investissements directs suisses à l'étranger ont marqué un net recul.

\section{$\square$ Rapport 2003 de la CNUCED sur l'investissement dans le monde}

Après une période de forte expansion qui avait duré près de deux décennies, les investissements directs étrangers (IDE) ont régressé pour la deuxième année consécutive et se chiffraient en 2002 à 651 milliards de dollars. L'état des IDE à l'échelle planétaire se monte ainsi à 7123 milliards de dollars. L'afflux d'IDE dans l'ensemble des pays en développement a régressé de 209 milliards en 2001

8 Dean Baker, Monique Morrissey, When Rivers Flow Upstream: International Capital Movements in the Era of Globalization, Briefing Paper, Washington, Centre for Economic and Policy Research (CEPR), 22 March 2003, <www.cepr.net/capital_flows.htm>.

9 UNCTAD (CNUCED), Trade and Development Report 2003 : Capital Accumulation, Growth and Structural Change, Geneva and New York, UN, 2003, tableau 2.2, <www.unctad.org>.

10 HM Treasury/Department for International Development (UK), International Finance Facility, London, January 2003,<www.hm-treasury.gov.uk/documents/international_issues/int_gnd_intfinance.cfm>. 
à 162 milliards en 2002. Selon la CNUCED, l'état des IDE dans les pays en développement totalisait 2340 milliards de dollars en $2002^{11}$ (tableau 29). Parmi les pays en développement et en transition, seuls quelques pays de l'Est et d'Extrême-Orient ont pu accroître leurs afflux en IDE.

La CNUCED attribue cette régression des flux d'IDE au manque de perspectives conjoncturelles et au ralentissement des privatisations dans de nombreux pays en développement. Le nombre de fusions et d'absorptions a considérablement diminué.

Tableau 29: Investissements directs étrangers: par régions ou pays, investissements entrants $(1998,2000-2002)$ et état $(1995,2002)$ (en milliards de dollars)

\begin{tabular}{|c|c|c|c|c|c|c|}
\hline \multirow[b]{2}{*}{ Région/Pays } & \multicolumn{4}{|c|}{ Investissements entrants } & \multicolumn{2}{|c|}{ Etat des IDE } \\
\hline & 1998 & 2000 & 2001 & 2002 & 1995 & 2002 \\
\hline Pays industrialisés & 472.0 & 1121.0 & 589.0 & 460.0 & 2041.0 & 4595.0 \\
\hline Europe & 263.0 & 710.0 & 401.0 & 384.0 & 1214.0 & 2780.0 \\
\hline Suisse & 8.9 & 19.3 & 8.9 & 9.3 & 57.0 & 118.0 \\
\hline Amerique du Nord & 197.0 & 381.0 & 173.0 & 51.0 & 659.0 & 1573.0 \\
\hline Japon & 3.2 & 8.3 & 6.2 & 9.3 & 34.0 & 60.0 \\
\hline Pays en dévelop./transition & 191.0 & 246.0 & 209.0 & 162.0 & 920.0 & 2340.0 \\
\hline Amérique latine/Caraïbes & 82.0 & 95.0 & 84.0 & 56.0 & 201.0 & 762.0 \\
\hline Asie/Pacifique & 100.0 & 142.0 & 107.0 & 95.0 & 641.0 & 1407.0 \\
\hline Sud/Sud-Est asiatique & 90.0 & 139.0 & 98.0 & 89.0 & 583.0 & 1305.0 \\
\hline Asie centrale & 3.0 & 1.9 & 4.0 & 4.0 & 4.0 & 25.0 \\
\hline Europe centrale/de l'Est & 22.0 & 26.0 & 25.0 & 29.0 & 40.0 & 188.0 \\
\hline Afrique & 8.9 & 8.5 & 18.8 & 11.0 & 77.0 & 171.0 \\
\hline Afrique du Nord & 2.9 & 3.1 & 5.5 & 3.5 & 26.0 & 48.0 \\
\hline Afrique subsaharienne & 6.0 & 5.4 & 13.3 & 7.5 & 51.0 & 123.0 \\
\hline Pays en dévelop. les plus pauvres & 4.6 & 3.4 & 5.6 & 5.2 & 16.0 & 46.0 \\
\hline Total & 686.0 & 1393.0 & 824.0 & 651.0 & 3002.0 & 7123.0 \\
\hline
\end{tabular}

Source: UNCTAD, World Investment Report 2003, tableaux B.1 et B.3.

Sur les 650 milliards de dollars investis en 2002, 600 milliards provenaient des pays industrialisés. Les investisseurs suisses y ont contribué à hauteur de 11,8 milliards de dollars (17,3 milliards en 2001 ; 44,7 milliards en 2000). A peine 50 milliards de dollars en IDE avaient pour origine les pays en développement et en transition (Amérique latine 5,8 milliards; Asie 3 milliards; Europe de l'Est 4 milliards; Afrique 0,17 milliard).

Le premier bénéficiaire parmi les pays en développement a été la Chine avec 50,7 milliards de dollars; c'est un des rares pays à avoir obtenu en 2002 davantage d'IDE que l'année précédente (46,8 milliards). Totalisant un état de 448 milliards de dollars en IDE, la Chine en compte désormais un volume presque équivalent à celui de l'Allemagne et de l'Angleterre. Si l'on y ajoute les 433 milliards de dollars investis à Hongkong, la Chine occupe même le deuxième rang derrière les Etats-Unis.

Les IDE effectués en 2002 ont totalisé 16,6 milliards de dollars au Brésil, 13,7 milliards à Hongkong, 13,6 milliards au Mexique, 9,3 milliards en Tchéquie

11 UNCTAD (CNUCED), World Investment Report 2003. FDI Policies for Development: National and International Perspectives, tableaux B.1, New York and Geneva, UNO, 2003, <www.unctad.org/wir>. 
et 7,7 milliards à Singapour. A titre de comparaison, les pays les plus pauvres (PMA) ont obtenu ensemble 5,2 milliards de dollars. Neuf dixièmes des IDE dans les pays en développement se concentrent toujours sur dix pays seulement.

Les IDE ont régressé alors même que l'on assouplissait encore davantage les dispositions légales relatives aux importations de capitaux. Pas moins de 70 pays ont pris en 2002 un total de 236 mesures visant à supprimer des obstacles dans ce domaine. Par ailleurs, 76 pays ont conclu 82 nouveaux accords bilatéraux de protection des investissements. Fin 2002, il y avait 2181 accords de ce genre en vigueur. Actuellement, $22 \%$ des IDE dans les pays en développement sont couverts par des accords bilatéraux. De plus, 64 Etats ont passé 68 nouveaux accords en matière de double imposition, dont le total atteint désormais 2256. Ces accords couvrent $57 \%$ des IDE effectués dans des pays en développement.

Le rapport 2003 sur l'investissement dans le monde souligne qu'il est essentiel pour les pays en développement de trouver un équilibre entre d'une part le maintien de conditions qui leur permettent d'élaborer une politique d'investissement autonome, et d'autre part la souplesse nécessaire pour tirer le meilleur parti possible de leurs accords.

\section{$\square$ Investissements directs de la Suisse}

La Banque nationale suisse indique que nos investissements directs à l'étranger se sont chiffrés à 16 milliards de francs en 2002, soit la moitié du chiffre atteint l'année précédente. Le recul est de $80 \%$ par rapport à 2000, année du maximum jamais réalisé. Cette désaffection a touché les pays en développement et les pays émergents bien davantage que les nations industrialisées (tableau 30) ${ }^{12}$.

Les investissements directs de l'étranger en Suisse ont eux aussi régressé dans de fortes proportions, passant de 32,5 milliards de francs en 2000 (maximum absolu) à seulement 4,5 milliards en 2002.

Plus que l'année précédente, l'économie suisse a concentré ses investissements dans quelques pays d'Europe de l'Est et d'Asie. C'est ainsi que les IDE helvétiques à Singapour ont dépassé le milliard de francs en 2002. Les entreprises suisses ont par contre désinvesti 28 millions de francs en Chine, mais le volume de leurs IDE dans ce pays reste considérable puisqu'il se chiffre à 2,1 milliards de francs. La BNS n'a pas fait de commentaires sur les motifs des désinvestissements massifs opérés en Amérique centrale et du Sud et (moins brutalement) en Afrique. Cela peut s'expliquer par une diminution des nouveaux crédits ou par le retrait d'anciens crédits à des filiales, ou encore par une baisse des réinvestissements (rapatriement des bénéfices) ou des nouveaux investissements.

12 Banque nationale suisse (BNS), La balance suisse des paiements 2002, Zurich, août 2003, $<$ www.snb.ch/f/publikationen/publi.html $>$. Voir aussi les publications trimestrielles, toutefois dépourvues d'indications sur la répartition géographique. 
Tableau 30: Investissements directs de la Suisse à l'étranger, 2000-2002 (en millions de francs)

\begin{tabular}{|c|c|c|c|c|c|c|}
\hline \multirow[t]{2}{*}{ Région } & \multicolumn{3}{|c|}{ Capitaux exportés } & \multicolumn{3}{|c|}{ Capitaux importés } \\
\hline & 2000 & 2001 & $2002^{a}$ & 2000 & 2001 & $2002^{\mathrm{a}}$ \\
\hline Pays industrialisés & 63693 & 22840 & 13591 & 3155 & 14701 & 4663 \\
\hline Europe centrale/de l'Est & 1095 & 1583 & 1708 & & & \\
\hline Pays émergents & 2014 & 802 & 213 & 814 & 181 & 12 \\
\hline Asie & 838 & 359 & 959 & . & . & \\
\hline Amérique centrale/du Sud & 1177 & 443 & -747 & & & \\
\hline Pays en développement & 9739 & 7239 & 1890 & 351 & 162 & -133 \\
\hline Asie & 251 & 630 & -10 & 20 & 22 & -222 \\
\hline Amérique centrale/du Sud & 9233 & 6535 & 1965 & 322 & 136 & 85 \\
\hline Afrique & 255 & 74 & -65 & 9 & 5 & 5 \\
\hline Tous les pays & 75446 & 30881 & 15693 & 32519 & 15045 & 4542 \\
\hline
\end{tabular}

Source: Banque nationale suisse, La balance suisse des paiements 2002, Zurich, août 2003, <www.snb.ch/f/publikationen/publi. html>.

a Chiffres provisoires.

\subsection{Endettement extérieur}

L'endettement des pays en développement s'est quelque peu alourdi en 2002. L'initiative en faveur des PPTE progresse plus lentement que prévu. Le débat sur le problème que pose la viabilité des dettes s'est intensifié.

\section{$\square$ Tendances}

L'endettement global des pays en développement et en transition a légèrement augmenté en 2002 et atteint désormais 2384 milliards de dollars (tableau 31). Seules les dettes privées sans garantie de l'Etat et les dettes à court terme ont diminué par rapport à l'année précédente.

Tableau 31 : Endettement des pays en développement et en transition (en milliards de dollars)

\begin{tabular}{|c|c|c|c|c|c|}
\hline & 1980 & 1990 & 2000 & 2001 & 2002 \\
\hline Endettement à long terme & 433.0 & 1155.0 & 1968.0 & 1908.0 & 1943.0 \\
\hline dont public et privé avec garantie de l'Etat & 365.0 & 1094.0 & 1434.0 & 1394.0 & 1435.0 \\
\hline public & 172.0 & 595.0 & 822.0 & 791.0 & 826.0 \\
\hline multilatéral & 47.0 & 204.0 & 333.0 & 339.0 & 365.0 \\
\hline - dont crédits à taux préférentiels & 18.0 & 69.0 & 129.0 & 130.0 & 144.0 \\
\hline bilatéral & 125.0 & 391.0 & 489.0 & 453.0 & 461.0 \\
\hline - dont crédits à taux préférentiels & 90.0 & 240.0 & 248.0 & 232.0 & 244.0 \\
\hline privé avec garantie de l'Etat & 193.0 & 499.0 & 612.0 & 603.0 & 609.0 \\
\hline privé sans garantie & 68.0 & 60.0 & 535.0 & 513.0 & 508.0 \\
\hline Crédits du FMI & 11.5 & 34.6 & 58.4 .0 & 75.2 .0 & 95.5 \\
\hline Crédits à court terme & 135.0 & 232.0 & 336.0 & 349.0 & 345.0 \\
\hline Endettement cumulé & 579.0 & 1422.0 & 2363.0 & 2332.0 & 2384.0 \\
\hline
\end{tabular}

Source:WB, Global Development Finance 2003, vol. 2, Washington, 2003, <www.worldbank.org/prospects/gdf2003>.

Les indices d'endettement (rapport dette/exportations de biens et de services et rapport service de la dette/exportations) ont marqué en 2002, presque partout, une légère amélioration depuis l'année précédente (tableau 32). Seule l'Amérique du Sud a vu son endettement croître quelque peu par rapport aux exportations. D’une manière générale cependant, l'endettement reste lourd puisqu'il 
représente toujours $39 \%$ du PNB en moyenne. En Amérique du Sud, ce rapport a passé de 41,5\% en 2001 à 48,2\%. Il a également augmenté un peu dans les pays du Moyen-Orient et d'Afrique du Nord, tandis qu'il diminuait légèrement dans toutes les autres régions de la planète.

Tableau 32: Indices d'endettement, 1990, 2000-2002

\begin{tabular}{lrrrrrrrrr}
\hline Catégories de pays & $\mathbf{d} / \mathbf{e x}^{\mathbf{a}}$ & & & & & $\mathbf{s d} / \mathbf{e x}^{\mathrm{b}}$ & & \\
\hline & $\mathbf{1 9 9 0}$ & $\mathbf{2 0 0 0}$ & $\mathbf{2 0 0 1}$ & $\mathbf{2 0 0 2}$ & & $\mathbf{1 9 9 0}$ & $\mathbf{2 0 0 0}$ & $\mathbf{2 0 0 1}$ & $\mathbf{2 0 0 2}$ \\
\hline Tous les pays en dévelop. & 170.8 & 119.4 & 118.5 & 112.8 & 18.7 & 19.3 & 19.2 & 16.2 \\
\hline Asie de l'Est/Pacifique & 135.2 & 77.2 & 79.9 & 71.1 & 17.8 & 11.4 & 12.1 & 10.9 \\
\hline Europe/Asie centrale & 306.3 & 121.9 & 114.6 & 110.1 & 17.1 & 18.4 & 21.7 & 18.0 \\
\hline Amérique latine/Caraïbes & 254.5 & 168.4 & 170.5 & 173.6 & 24.4 & 38.6 & 35.5 & 29.6 \\
\hline Moyen-Orient/Afr. du Nord & 118.9 & 88.1 & 88.7 & 86.9 & 15.6 & 10.1 & 9.5 & 8.7 \\
\hline Asie du sud & 324.7 & 154.1 & 147.1 & 138.6 & 28.7 & 14.7 & 12.7 & 11.4 \\
\hline Afrique subsaharienne & 208.6 & 175.2 & 170.2 & 164.5 & 12.8 & 11.2 & 11.2 & 10.7 \\
\hline Pays à faible revenu & 293.5 & 181.3 & 176.6 & - & 22.8 & 15.8 & 14.4 & - \\
\hline Pays à revenu intermédiaire & 145.0 & 108.1 & 107.9 & - & 17.8 & 19.9 & 20.1 & - \\
\hline
\end{tabular}

Source:WB, GDF 2003, vol. 2.

a d/ex = rapport dette/exportations de biens et services.

${ }^{\mathrm{b}} \mathrm{sd} / \mathrm{ex}=$ rapport service de la dette/exportations de biens et services.

\section{$\square$ L'initiative PPTE}

En septembre 2003, après que la République démocratique du Congo eut fait le pas, 27 des 38 pays classés initialement dans la catégorie des pays pauvres très endettés (PPTE) avaient atteint le point de décision. Huit pays ont atteint le point d'achèvement (nouveaux: Mali et Bénin) ${ }^{13}$. L'initiative de désendettement internationale lancée par le FMI et la Banque mondiale a pour but d'effacer en partie ce que les pays les plus pauvres et les plus endettés doivent à l'ensemble de leurs créanciers, de telle sorte que le poids de leur endettement ne dépasse plus la limite du supportable. Les pays bénéficiaires de ces allégements s'engagent en contrepartie à suivre une politique macroéconomique équilibrée.

[D] Annuaire 2003, $\mathrm{n}^{\text {0 }}$ 1, pp. 106 sqq.; 2002, pp. 137 sqq.; 2001, pp. 149 sqq.;2000, pp. 129 sqq.

Le FMI et la Banque mondiale reconnaissent que le processus nécessaire pour aboutir au point d'achèvement prend davantage de temps que prévu initialement. Ces institutions expliquent une telle lenteur par la peine qu'ont les pays concernés à respecter leurs programmes de stabilité macroéconomique. L'élaboration des stratégies de réduction de la pauvreté (SRP) est elle-même longue et laborieuse. Ethiopie, Guyane, Nicaragua, Niger, Rwanda et Sénégal seront les prochains pays à atteindre le point d'achèvement. Les plus grands obstacles que rencontrent les pays non encore qualifiés sont des conflits internes ainsi que des arriérés de paiement aux institutions multilatérales. La Suisse a plaidé pour que la qualité du programme ne souffre pas des efforts entrepris en vue d'accélérer la concrétisation de l'initiative PPTE ${ }^{14}$.

Les pays qui ont atteint le point de décision verront leur encours de la dette à 2002 (valeurs actuelles nettes) réduit de deux tiers, de 77 à 32 milliards de dollars, ce

13 IMF/WB, Heavily Indebted Poor Countries (HIPC) Initiative: Status of Implementation, Washington, 12 September 2003, <www.imf.org/external/np/hipc/2003/status/091203.htm>.

14 IMF, Executive Director for Switzerland (and others), Activity Report 2003, Washington, September 2003, p. 26. 
qui entraîne une baisse des indices de l'endettement: pour les 27 pays qui ont atteint le point de décision, le taux du service de la dette est ramené en moyenne à 9,9\% (contre 15,7\% en 1998-1999). Les dépenses de lutte contre la pauvreté dans ces 27 pays ont atteint en 2002 près de quatre fois le montant du service de la dette. L'afflux de capitaux dans ces pays a continué d'augmenter, ce qui a conduit le FMI et la Banque mondiale à admettre que les mesures de désendettement se sont dans une large mesure ajoutées au reste de l'aide.

Par ailleurs, FMI et Banque mondiale ont souligné plus clairement que jamais que l'initiative PPTE ne constitue pas en elle-même une garantie de viabilité de la dette. FMI, Banque mondiale, gouvernements, spécialistes et ONG ont consacré au cours de l'année 2003 un certain nombre d'études et de séminaires à cette problématique. On admet d'une manière générale qu'il faudra adopter à l'avenir une attitude plus prévoyante. Ce dont on a besoin, ce sont des stratégies de financement clairement définies, une gestion maîtrisée des crédits et de l'endettement, des analyses de risques pour les chocs externes et, surtout, l'examen des répercussions prévisibles sur les objectifs du millénaire pour le développement ${ }^{15}$.

En dépit de prévisions incertaines, FMI et Banque mondiale estiment toujours que les coûts de l'initiative PPTE restent finançables, même si des opérations de topping up sont nécessaires pour compenser les difficultés imprévues que peuvent rencontrer certains pays. La Suisse a toutefois mis en garde contre une générosité excessive à cet égard tant que les fonds correspondants n'ont pas été débloqués. Le Conseil fédéral a décidé en décembre 2002 d'ajouter aux 90 millions de francs déjà versés 50 millions destinés à financer le fonds PPTE de la Banque mondiale.

Le Département d'évaluation des opérations (Operation Evaluation Department, OED), instance indépendante de la Banque mondiale, a souligné dans une de ses études que l'initiative PPTE constitue un pas important mais insuffisant pour résoudre le problème de l'endettement. Il convient de préciser les objectifs, de rendre la méthode utilisée pour y parvenir plus transparente, d'en accroître l'efficacité et de renforcer l'aspect «lutte contre la pauvreté ${ }^{16}$.

Les ONG se montrent, quant à elles, plus critiques à l'égard de l'initiative PPTE. Un «rapport fantôme» observe que l'initiative a pris énormément de retard sur son calendrier, que les créanciers n'y contribuent pas tous dans les mêmes proportions, que les institutions de Bretton Woods devraient annuler entièrement leurs dettes. On critique également le principe de la conditionnalité ; les objectifs du millénaire ne pourront pas être atteints dans ces conditions ${ }^{17}$.

15 Exemple représentatif d'une littérature abondante: IMF/WB, Report on Joint Bank-Fund Workshop on Debt Sustainability in Low-Income Countries, Washington D.C., 11-12 September 2003, avec une bibliographie, <www.imf.org/external/np/pdr/sustain/2003/100903.htm>. Egalement intéressant: travail de diplôme d'un ancien collaborateur du service de désendettement de la Communauté de travail des œuvres d'entraide, en collaboration avec la New Economic Foundation/Jubilee 2000 : Stefan Hochhuth, The Impact of Debt Relief: Does the HIPC Initiative Help in Meeting the Millennium Development Goals ?, Discussion Paper, London, 14 October 2003 (manuscrit non publié).

16 WB, OED, The Heavily Indebted Poor Countries (HIPC) Initiative: An OED Review, report $\mathrm{n}^{\mathrm{o}}$ 25160, Washington, 20 February 2003, <www.worldbank.org/oed>.

17 Romilly Greenhill, Elena Sisti, Real Progress Report on HIPC, NEF/Jubilee, London, September 2003, <www.jubileeresearch.org >. 


\section{$\square$ Dettes publiques}

Les dettes publiques de nombreux pays émergents à l'égard de leurs créanciers intérieurs et étrangers sont-elles encore viables? FMI et Banque mondiale signalent leur préoccupation croissante face à l'augmentation rapide de ces dettes. Quantité de gouvernements ont profité de la baisse des taux d'intérêt pour s'endetter sur le marché financier plus vite et davantage que ne le permettrait leur base fiscale. Les institutions de Bretton Woods ont publié fin 2002 des directives concernant la gestion des dettes publiques. Le numéro de septembre du World Economic Outlook publié par le FMI consacre son dossier à cette problématique. Il estime qu'un fort endettement accroît le risque de crises financières et limite la flexibilité de la politique fiscale. Le FMI préconise des correctifs dans la politique des recettes aussi bien que dans celle des dépenses ${ }^{18}$.

\section{$\square$ Réaménagement de dettes publiques}

Les gouvernements de six pays - Argentine, Gabon, Indonésie, Madagascar, Moldavie et Nauru - se sont vus en 2002 dans l'impossibilité d'honorer leur service de la dette. Cela en porte le nombre à 28 , un total qui n'avait plus été atteint depuis $1992^{19}$. Contrairement à ce qui était le cas autrefois, les gouvernements s'endettent non plus tellement auprès des banques que sous la forme d'emprunts obligataires directement souscrits sur les marchés financiers. La Banque mondiale reconnaît que le Pakistan, l'Ukraine et l'Argentine ont su faire de bonnes restructurations obligataires ces dernières années, mais estime qu'il existe un réel danger de restructurations désordonnées. Elle plaide ainsi pour une meilleure gestion de la crise (clauses collectives, mécanisme pour les créances irrécouvrables) ${ }^{20}$.

Le Club de Paris a conclu en 2002, avec dix pays, des accords portant sur le rééchelonnement de dettes multilatérales pour un total de 20 milliards de dollars. Le plus gros débiteur était la République démocratique du Congo (9 milliards). Jordanie mise à part, il s'agissait exclusivement de pays à faible revenu. Huit d'entre eux s'inscrivent dans l'initiative PPTE. La Suisse a participé aux négociations de réaménagement avec trois pays, à savoir l'Indonésie, la Jordanie et la République démocratique du Congo.

En 2003, le Club de Paris avait mené à bien jusqu'à fin novembre de nouveaux réaménagements de dettes multilatérales pour trois pays ${ }^{21}$. Le Mali a bénéficié d'annulations de dettes chiffrées à 70 millions de dollars, et le Bénin pour un total de 60 millions. L'Equateur s'est vu accorder le rééchelonnement de 81 millions de dollars sur quinze ans plus trois ans à titre de délai de carence.

Sur la base des résolutions prises par le sommet du G8 à Evian, le Club de Paris a adopté en octobre 2003 de nouvelles directives concernant les pays débiteurs qui n'appartiennent pas à la catégorie PPTE. Il s'agit fondamentalement de mieux prendre en considération la situation particulière de chaque pays. Ceux

\footnotetext{
18 IMF, World Economic Outlook: Public Debt in Emerging Markets, Washington, September 2003, $<$ www.imf.org/external/pubs/ft/weo/2003/02>. Et aussi : IMF/WB, Guidelines for Public Debt Management, 21 November 2002, <www.imf.org/external/np/mae/pdebt/2002/eng/102402.htm>.

19 WB, GDF 2003, vol. 1, p. 56.

20 Voir plus bas la partie 7.4.2.

21 Club de Paris, divers communiqués, <www.clubdeparis.org $>$.
} 
dont les dettes sont considérées comme non viables par le FMI et le Club de Paris devraient notamment pouvoir compter sur des remises plus importantes.

Cette décision a été prise entre autres dans le contexte de la dette irakienne, qui a occupé le Club de Paris à plusieurs reprises. En juillet 2003, celui-ci a estimé les créances de ses pays membres à 21'018 milliards de dollars. La part de la Suisse se montait à 117,5 millions. L'endettement total de l'Irak dépasse 120 milliards de dollars.

La Suisse a conclu en 2002 des accords bilatéraux de ce type avec la République fédérale de Yougoslavie (de l'époque) (296 millions de francs), le Cameroun (7 millions), le Nigeria (255 millions), le Pakistan (119 millions) et l'Indonésie (143 millions), sur la base des accords multilatéraux du Club de Paris. Les accords avec le Nigeria et le Pakistan ne comportent pas de réduction de dette. D'importantes remises de dettes ont été accordées au Cameroun $(90 \%)$ et à la République fédérale de Yougoslavie (60\%). Un accord de désendettement (10 millions de francs) a en outre été conclu avec le Cameroun ${ }^{22}$. Pour 2003, on avait enregistré à fin novembre un seul nouvel accord bilatéral avec la Jordanie, portant sur le rééchelonnement de dettes totalisant 18 millions de francs.

La Suisse a procédé dans le cadre de l'initiative PPTE à un désendettement partiel du Sierra Leone à hauteur de 8,8 millions de francs. Fin 2003, elle a entièrement abandonné ses créances restantes totalisant 31 millions de francs à l'égard de la République démocratique du Congo. Elle a en outre versé 5 millions de dollars à titre de participation à une action concertée d'annulation des dettes en souffrance du Congo envers la Banque africaine de développement.

\section{$\square$ Dettes commerciales}

En 2002, trois pays - Argentine, Gabon, Moldavie - n'étaient plus en mesure d'honorer leurs dettes à l'égard des créanciers privés. L'Indonésie a restructuré dans le cadre du Club de Londres des crédits bancaires totalisant 1,5 milliard de dollars. Les Seychelles, de leur côté, ont pu réaménager pour 70 millions de dollars de dettes à l'égard de banques commerciales.

L'AID, filiale de la Banque mondiale, n'a pas recouru en 2002 à son Fonds de réduction de la dette. En 2003, la Tanzanie et le Cameroun ont pu solliciter ce fonds avec l'aide de la Suisse et de l'Allemagne. Les négociations engagées avec le Mozambique n'avaient pas encore abouti à fin 2003.

\section{$\square$ Autres mesures de désendettement prises par la Suisse}

La Suisse continue de soutenir le Debt Management Capacity Building Program $^{23}$ ainsi que les programmes de la CNUCED. Le service de désendettement de la Communauté de travail a poursuivi les opérations de clôture des derniers fonds de contrepartie. Le mandat du service de désendettement a été complété par des tâches spécifiques portant sur la politique internationale de développement et les DSRP dans les PPTE24.

22 Conseil fédéral, Raport sur la politique économique extérieure 2002, 15 janvier 2003, <www.seco. admin.ch/publikationen>.

23 Responsable: Debt Relief International, <www.dri.org.uk>.

24 Voir plus bas, dans la partie 7.4.1, «Le DRSP - un principe qui s'impose peu à peu ». 


\subsubsection{Les institutions de Bretton Woods (IBW) dans le contexte international}

Les conférences internationales de ces dernières années, et les objectifs du millénaire qui en sont résultés, confirment la responsabilité collective de tous les partenaires concernés par le processus de développement. En particulier, le consensus de Monterrey définit un cadre qui spécifie acteurs et actions à entreprendre. Cela contraint les institutions de Bretton Woods à des réformes: elles doivent définir plus clairement leurs fonctions, préciser la répartition de leurs tâches et mieux coopérer avec leurs autres partenaires.

\section{$\square$ Le nouveau partenariat pour le développement}

Les nouveaux instruments instaurés ces dernières années, comme les stratégies de réduction de la pauvreté et le Cadre de développement intégré (Comprehensive Development Framework, $C D F$ ), se sont en quelque sorte cristallisés en un nouveau modèle de développement. Le regard de la coopération internationale se focalise désormais sur les pays directement concernés. Chaque pays en développement est tenu de produire, sur le mode participatif, un diagnostic de sa situation, d'élaborer des stratégies et des politiques portant en particulier sur la réduction de la pauvreté (document de stratégie pour la réduction de la pauvreté, $D S R P)^{25}$.

Les donateurs multilatéraux et bilatéraux doivent axer leurs programmes de développement sur ces DSRP spécifiques à chaque pays, veiller à un financement approprié, harmoniser et coordonner leurs politiques et leurs méthodes. Une rencontre de haut niveau a eu lieu sur ce thème à Rome en février 2003; des représentants des IBW, de l'ONU, des banques de développement régionales, de l'OCDE et de nombreux gouvernements y ont adopté la Déclaration de Rome $^{26}$. La Suisse y a également participé. FMI et Banque mondiale ont précisé à cette occasion la manière dont les deux institutions se répartissent les tâches ainsi que les formes de coopération adoptées. L'une et l'autre ont explicité leur politique et leur stratégie, notamment à l'égard des pays pauvres et dans la perspective des objectifs du millénaire.

Les IBW ont participé dans le cadre des Nations unies au suivi du processus de Monterrey (Financing for Development, FfD). Juste après leur session de printemps s'est tenue pour la seconde fois une réunion de haut niveau entre le Conseil économique et social (ECOSOC), les IBW et l'OMC ${ }^{27}$. Il s'agissait d'une part d'améliorer la cohérence du travail accompli par les différentes organisations internationales, d'autre part de trouver des méthodes pour mieux mesurer les efforts nationaux et internationaux entrepris pour mettre en œuvre les résolutions de Monterrey concernant les objectifs du millénaire. Et fin octobre a eu lieu, dans le cadre des Nations unies, une discussion de haut rang

25 Voir WB, Executive Director for Azerbaijan, Kyrgyz Republic, Poland, Serbia and Montenegro, Switzerland, Tajikistan, Turkmenistan, Uzbekistan, Report to the Governors for the Annual Meetings, September 2003, Washington.

26 Rome Declaration on Harmonization: High Level Forum on Harmonization, Rome, 24-25 February 2003, <www1.worldbank.org/harmonization/romehlf>.

27 Voir <www.un.org/esa/coordination/ecosoc/bwi2003.htm>. 
sur le financement du développement. Quant aux ONG suisses, Action de carême y a participé dans le cadre de son organisation internationale (Coopération internationale pour le développement et la solidarité, CIDSE). Action de carême avait auparavant écrit aux autorités suisses pour les prier de soutenir le souhait exprimé par des ONG internationales de créer un groupe de travail qui se consacrerait aux questions d'endettement au sein de l'ONU².

\section{$\square$ Accorder davantage de poids aux pays en développement dans le cadre des IBW}

Rendre les IBW plus démocratiques est une vieille revendication, qui a cependant pris un nouvel élan après Monterrey. Le FMI et la Banque mondiale devaient proposer dans des rapports ad hoc des moyens de faire en sorte que les pays en développement et en transition aient davantage voix au chapitre. Des documents à ce sujet ont été présentés à la session de printemps aussi bien qu'à l'assemblée annuelle ${ }^{29}$. Mais il n'y a pas eu de réformes substantielles jusqu'à présent. Les bureaux des administrateurs africains, notamment, sont mieux dotés en ressources humaines et financières. Une révision des droits de vote est à l'étude, mais se heurte au refus des Etats-Unis. La Suisse a soutenu les mesures prises sur le plan du personnel, mais se contentera pour le reste de participer à la suite du débat relatif à la révision des droits de vote.

\section{$\square$ Le DSRP - un principe qui s'impose peu à peu}

Le principe d'élaborer, sur le mode participatif, des documents de stratégie pour la réduction de la pauvreté est désormais chose admise. Mais en dépit des progrès réalisés, ni les processus ni les résultats ne peuvent être qualifiés de francs succès - comme le montrent d'une part les habituels rapports semestriels publiés conjointement par le FMI et la Banque mondiale, et d'autre part une quantité d'études, de conférences et de séminaires ${ }^{30}$.

Un total de 32 pays avaient réalisé des documents de stratégie de réduction de la pauvreté (DSRP) jusqu'à septembre 2003 et ces documents étaient en voie d'élaboration dans 21 autres pays. Selon une estimation commune du FMI et de la Banque mondiale, ce processus a pris plus de temps que prévu initialement. L'engagement réel de certains gouvernements est mis en doute. D'autres documents stratégiques n'ont pas réussi à trouver le juste milieu entre ambition et réalisme. Des lacunes se manifestent dans l'établissement de priorités et la concrétisation des stratégies au niveau budgétaire. Et, finalement, les donateurs n'ont toujours pas su adapter leur aide aux stratégies nationales.

Les ONG, quant à elles, déplorent surtout que les DSRP n'aient pas fait renoncer aux vieux principes de la stabilité macroéconomique et de l'ajustement

28 Voir <www.un.org/esa/ffd/ffd1003ga-hileveldialogue.htm>. L'échange de lettres avec Action de carême n'a pas été publié.

$29<$ <ww.worldbank.org/annualmeetings $>$.

30 La littérature sur les DSRP est très riche. FMI et Banque mondiale ont leurs propres sites Internet: <www.worldbank.org/poverty/strategies/index.htm > et <www.imf.org/external/np/exr/facts/prsp.htm>. On y trouve aussi des rapports détaillés sur leur avancement. CNUCED, OIT et banques de développement régionales publient aussi des études sur ce sujet. Du côté des ONG, voir surtout les sites d'Eurodad et du projet de réforme des institutions de Bretton Woods : <www.eurodad.org $>$ et $<$ www. brettonwoodsproject.org $>$. 
structurel. Le poids des IBW reste excessif dans l'élaboration des SRP; on se montre souvent sélectif dans le choix des organisations participantes de la société civile, le rôle joué par les parlements nationaux est généralement négligeable. Il y a un fossé entre ambition et réalité des SRP. Et il faut se souvenir que c'est la concrétisation d'une stratégie qui en fait la valeur.

Quant à la Suisse, la DDC aussi bien que le seco soutiennent le processus des SRP. La DDC a fait évaluer par une instance indépendante ses contributions à ce processus dans quatre pays partenaires. L'examen a également porté sur les influences subies lors de l'élaboration et de la réalisation du programme de la DDC $^{31}$. Le rapport d'évaluation a recommandé notamment une application plus énergique du principe des SRP au sein de la DDC et du seco, davantage de coopération avec d'autres pays donateurs ainsi qu'un soutien actif du processus dans les pays partenaires, notamment au niveau de la société civile. Le seco a préparé, dans le cadre d'un projet pilote, une participation au fonds fiduciaire multilatéral pour les SRP, et prévoit de soutenir des petits projets bilatéraux.

Dans le cadre de son mandat élargi, le service de désendettement de la Communauté de travail a réalisé pour le seco et la DDC deux études concernant la participation du secteur civil au processus de SRP au Ghana et au Burkina Faso ${ }^{32}$. Enfin, un colloque réunissant la DDC et des ONG en juin 2003 a examiné la problématique «participation» dans la mise en œuvre des DSRP.

\section{$\square$ Commerce et développement}

Le FMI aussi bien que la Banque mondiale ont accordé une importance centrale à la question «commerce et développement». Les deux institutions ont vigoureusement réclamé une poursuite de la libéralisation du commerce et le renforcement du système du commerce international. Peu avant la rencontre ministérielle de l'OMC à Cancún, MM. Wolfensohn, président de la Banque mondiale, et Köhler, directeur général du FMI, ont adressé à l'OMC et à ses pays membres une lettre commune dans laquelle ils soulignent l'importance du commerce pour la prospérité future du monde, tout en critiquant les pays industrialisés pour leur attitude protectionniste. A mi-décembre 2003, MM. Köhler et Wolfensohn ont à nouveau exhorté les nations industrialisées à lever leurs barrières douanières à l'égard des pays en développement et à mettre fin aux distorsions commerciales que provoquent leurs subventions aux exportations.

La Banque et le FMI ont préconisé une intégration de la politique commerciale dans les DSRP. Dans le cadre de son activité de surveillance, le FMI a résolu de se concentrer davantage sur les problèmes d'accès aux marchés que rencontrent les pays en développement, et de renforcer l'assistance technique dans ce domaine. Par ailleurs, le FMI est disposé à accorder des crédits transitoires pour compenser les difficultés de balance des paiements résultant des pertes de recettes consécutives aux suppressions de barrières douanières.

La Banque mondiale a soutenu les réformes entreprises par les pays en développement dans le domaine du commerce extérieur, en vue de renforcer leurs

31 DDC, SDC's Bilateral Engagement in the Poverty Reduction Stategy Paper (PRSP) Process: Evaluation, 2003/1, <www.deza.admin.ch>. Voir également <www.gersterconsulting.ch/docs/Synthesis_final. pdf $>$.

32 Ces deux études recommandent de promouvoir une meilleure participation de la société civile locale. Voir <www.swisscoalition.ch/english/pagesnav/T.htm $>$. 
capacités d'exportation. La publication annuelle Global Economic Prospects a consacré son édition 2004 à la question de savoir comment tenir les promesses faites dans le cadre du programme de Doha pour le développement ${ }^{33}$.

\section{Rapport au Parlement sur le rôle de la Suisse au sein des IBW}

La Commission de gestion $(\mathrm{CdG})$ du Conseil des Etats a examiné, avec l'aide d'experts externes, les objectifs, l'influence, les coûts et les avantages de l'adhésion de la Suisse aux institutions de Bretton Wood, et a publié en octobre 2003 un rapport sur ce sujet ${ }^{34}$. Ce rapport recommande au Conseil fédéral de réexaminer la répartition des tâches entre DDC et seco pour le Groupe de la Banque mondiale. Il préconise également de mieux harmoniser les positions helvétiques au sein de la Banque mondiale et du FMI. Et les prestations financières aussi bien que l'activité politique devraient faire l'objet d'une transparence accrue.

\subsubsection{Fonds monétaire international (FMI)}

Le FMI a continué d'accorder un volume élevé de crédits, tout en renforçant son dispositif de surveillance et de prévention des crises. Mais il n'a pas pu mettre en place un mécanisme international en vue de dépasser les situations d'insolvabilité.

\section{$\square$ Volume de crédits toujours élevé}

Au cours de son exercice clôturé à fin avril 2003³, le FMI a octroyé 22 crédits totalisant 30,5 milliards de DTS (droits de tirage spéciaux). Il n'a pas atteint le record des engagements faits l'année précédente (41,3 milliards de DTS), mais l'ensemble des crédits non remboursés atteignait le montant inégalé jusque-là de 73 milliards de DTS (tableau 33).

Les crédits versés ont dépassé les remboursements, de sorte que l'on compte un transfert net de 15 milliards de DTS. Le Brésil a obtenu le plus gros crédit jamais octroyé - 23 milliards de DTS -, suivi par l'Argentine, la Colombie et l’Uruguay. Plus de trois quarts des crédits engagés se sont concentrés sur le Brésil, l'Argentine et la Turquie. La Suisse a observé cette focalisation avec une inquiétude croissante.

Tableau 33: Crédits du FMI, 1999-2003 (en milliards de DTS, clôture au 30 avril)

\begin{tabular}{lrrrrr}
\hline & $\mathbf{1 9 9 9}$ & $\mathbf{2 0 0 0}$ & $\mathbf{2 0 0 1}$ & $\mathbf{2 0 0 2}$ & $\mathbf{2 0 0 3}$ \\
\hline Engagements & 29.4 & 22.9 & 14.3 & 41.3 & 30.6 \\
\hline Versements & 24.9 & 6.9 & 10.2 & 30.1 & 23.0 \\
\hline Remboursements & 11.1 & 23.6 & 11.8 & 20.0 & 7.8 \\
\hline Transferts nets & 13.8 & -16.7 & -1.6 & 10.1 & 15.2 \\
\hline Crédits non remboursés & 67.2 & 50.4 & 48.7 & 58.7 & 72.9 \\
\hline Obligations non honorées à l'échéance & 2.3 & 2.3 & 2.2 & 2.1 & 1.7 \\
\hline Nombre d'accords de prêt & 56.0 & 58.0 & 62.0 & 52.0 & 54.0 \\
\hline
\end{tabular}

Source: IMF, Annual Report 2003, Washington, 2003, <www.imf.org/external/pubs/ft/ar/2003/eng/index.htm>.

33 WB, Global Economic Prospects: Realizing the Developing Promise of the Doha Agenda, Washington, 2003, <www.worldbank.org/prospects/gep2004>.

$34 \mathrm{CdG}$ du Conseil des Etats, La Suisse comme membre des institutions de Bretton Woods, Berne, 14 octobre 2003, <www.parlament.ch/f/ed-pa-gpk-s-brettonwoods-d.pdf>.

35 IMF, Annual Report 2003, Washington, 2003, <www.imf.org/external/pubs/ft/ar/2003/eng/index.htm>. 


\section{$\square$ Surveillance et prévention de crises}

Le FMI a poursuivi la surveillance renforcée qu'il exerce sur les pays membres ainsi qu' aux échelons régional et global. Sa surveillance globale a pris la forme de discussions régulières au sein de son conseil d'administration. Le FMI a publié comme de coutume sa revue semestrielle World Economic Outlook (WEO). Son numéro d'avril 2003 a été consacré notamment aux répercussions sur le marché financier de l'éclatement de la bulle spéculative, à l'importance d'institutions fortes pour la croissance, ainsi qu'à des problèmes d'emploi et de marché du travail. L'édition de septembre 2003 a plus particulièrement analysé la forte progression de l'endettement public (interne et externe) de nombreux pays émergents. Pour les questions essentiellement financières, le FMI publie également la revue trimestrielle Global Financial Stability Report ${ }^{36}$.

Du côté des pays membres, il y a eu en tout 136 contrôles dans le cadre des consultations au titre de l'article 4 des statuts du FMI. La majeure partie de ces consultations ont été publiées de bon gré. Le conseil d'administration n'a pas pu se résoudre à rendre cette publication obligatoire.

Pour la Suisse, ces consultations ont eu lieu en février 2003 puis ont été discutées en mai au sein du conseil d'administration ${ }^{37}$. La Communauté de travail des œuvres d'entraide y a participé; elle est tombée d'accord avec la délégation du FMI que la Suisse, en dépit de difficultés budgétaires, aurait les moyens d'augmenter son aide publique au développement.

Le FMI a voué une attention particulière à ses grands débiteurs d'Amérique latine. L'Argentine a eu un certain succès dans ses efforts de stabilisation et a bénéficié en janvier 2003, grâce à l'insistance du G7, d'un programme d'appui à court terme du FMI qui revenait pratiquement à prolonger des dettes échues. La Suisse ${ }^{38}$ s'est abstenue de voter, a critiqué le fait que l'Argentine coopérait mal avec le FMI et tardait toujours à réaliser les réformes en suspens. Lors de l'assemblée annuelle de septembre, l'administrateur suisse et trois de ses collègues se sont également abstenus d'approuver le nouvel accord d'assistance pour trois ans assorti d'un crédit de 15 milliards de dollars. Jean-Pierre Roth, président de la Banque nationale suisse, a déclaré que l'on récompensait ainsi un pays qui n'avait pas de réformes concrètes à faire valoir et se montrait peu coopératif

36 IMF, World Economic Outlook: Growth and Institutions, Washington, April 2003, <www.imf.org/ external/pubs/ft/weo/2003/01> ; IMF, World Economic Outlook: Public Debt in Emerging Markets, Washington, September 2003, <www.imf.org/external/pubs/ft/weo/2003/02> ; IMF, Global Financial Stability Report, diverses publications, Washington, <www.imf.org/external/pubs/ft/gfsr/about.htm>. Publication d'un «WEO parallèle» par un collectif d'auteurs de Jubilee Research: Ann Pettifor (ed.), Real World Economic Outlook. The Legacy of Globalization: Debt and Inflation, Jubilee Research at the New Economics Foundation, Hampshire and New York, Palgrave Macmillan, 2003.

37 IMF, Switzerland-2003 Article IV Consultation: Concluding Statement, Bern, 24 February 2003, $<$ www.imf.org/external/np/ms/2003/022403.htm> ; DFF, Le FMI ne constate aucun déséquilibre fondamental dans l'économie suisse, communiqué de presse, 24 février 2003, <www.efd.admin.ch/ f/dok/medien/medienmitteilungen/2003/02/iwf.htm>. Par ailleurs: IMF, Switzerland: 2003 Article IV Consultation - Staff Report..., Country Report, nº 03/148, 2 June 2003, <www.imf.org/external/ pubs/cat/longres.cfm?sk=16579.0>.

38 Le Département fédéral des finances publie depuis le début de 2003 sur son site Internet sa newsletter aux commissions de politique étrangère du parlement. Il y donne des informations claires et substantielles sur les affaires en cours du FMI et sur la position suisse à cet égard: DFF, Newsletter concernant le FMI, <www.efv.admin.ch/f/internat/iwf_int/index.htm>. 
avec le $\mathrm{FMI}^{39}$. Fin novembre 2003, l'Argentine négociait toujours un accord de rééchelonnement avec ses créanciers privés.

Le Brésil, en revanche, a continué, sous son nouveau président, Lula da Silva, de répondre aux attentes du FMI. Mais sa très grande vulnérabilité, due notamment à un énorme endettement extérieur, a incité le FMI à reconduire début novembre 2003 les accords passés avec son plus gros débiteur. C'est ainsi que le Brésil obtient de nouveaux crédits à hauteur de 6 milliards de DTS, auxquels s'ajoutent 8 milliards de l'ancien accord pas encore mis à contribution. La Suisse s'est associée à ces décisions.

La Turquie a tout juste rempli les critères du FMI, et dû demander une prolongation de délai pour les remboursements. Les Etats-Unis lui accordent un crédit bilatéral de 5,8 milliards de dollars destiné à soutenir les réformes économiques et à atténuer les répercussions de la guerre en Irak. La Suisse a regretté les retards subis par le programme turc de réformes économiques.

Le FMI a poursuivi le renforcement de ses instruments de surveillance et de prévention des crises. Il s'agit en particulier d'améliorer la préparation des données économiques et la publication de celles-ci, et de systématiser les normes et codes reconnus sur le plan international. La Suisse a soutenu ces améliorations, mais voulait surtout que l'on se concentre sur les aspects d'ordre macroéconomique. Le FMI a par ailleurs perfectionné les instruments qui lui servent à surveiller la viabilité des dettes. Il a en revanche laissé s'éteindre à fin novembre 2003 les lignes de crédit contingent credit lines (CCL) créées par mesure de précaution après les crises financières de la fin des années 1990. Les CCL étaient censées offrir aux pays pratiquant une bonne politique économique des moyens financiers supplémentaires pour affronter des crises dont ils n'étaient pas responsables, mais elles n'ont jamais été mises à contribution. La Suisse également s'est opposée à leur maintien ${ }^{40}$.

\section{$\square$ Améliorer la réglementation pour surmonter les crises}

Le FMI a adopté des règles plus strictes concernant l'obtention de crédits par des pays confrontés à des crises de leur balance des paiements. Ses conditions sont entre autres un niveau d'endettement qui doit rester viable, la capacité de revenir au marché des capitaux privés, ainsi que la volonté politique et la faculté de réaliser le programme d'ajustement prévu par le FMI. La Suisse a soutenu ce projet, considérant que celui-ci favorise sélectivité, transparence et uniformité dans l'octroi de crédits.

La direction du FMI estime que si l'endettement d'un pays n'est pas viable, il faut disposer d'un système international de restructuration des dettes de l'Etat (mécanisme de restructuration de la dette souveraine, MRDS). Ses propositions, discutées durant plus d'une année, ont été brusquement gelées lors de la session de printemps 2003 après que les Etats-Unis eurent refusé de donner leur accord aux modifications nécessaires des statuts du FMI. Les banques privées internationales - dont les grandes banques suisses - se sont également opposées avec vigueur au principe du MRDS. Celui-ci devrait être remplacé par des

$39 \quad$ NZZ, 25. September 2003.

40 Fritz Zurbrügg (administrateur suisse auprès du FMI), «Was tun zur Stärkung der Krisenprävention. Der IMF braucht kein spezielles Kreditinstrument», NZZ, 29. Oktober 2003. 
clauses de consolidation collective incluses dans les contrats d'obligations. La Suisse a soutenu ces efforts, entre autres dans le cadre d'un rapport du G10 à ce sujet ${ }^{41}$.

Comme la plupart des pays européens, la Suisse aurait approuvé ce MRDS. Les propositions d'ONG internationales visant à créer un tribunal d'arbitrage n'ont bénéficié que d'un début d'intérêt ${ }^{42}$. Les efforts entrepris dans le cadre du suivi de la Conférence de Monterrey sur le financement du développement ne sont pas parvenus, jusqu'à présent, à faire de l'ONU l'instance compétente pour cette réglementation internationale en matière d'insolvabilité.

Annuaire 2003, $n^{\circ}$ 1, «Réforme du système financier international», pp. 113-114.

\section{$\square$ Le Bureau d'évaluation indépendant}

Le Bureau d'évaluation indépendant (IEO) créé en 2001 publie régulièrement des programmes de travail et des comptes rendus. Durant l'exercice écoulé, il a achevé et présenté trois rapports au conseil d'administration ${ }^{43}$. Fin 2003, des rapports étaient encore en voie d'élaboration concernant les stratégies de réduction de la pauvreté (SRP), le rôle du FMI en Argentine, ainsi que l'assistance technique.

Ces évaluations ont exercé une influence sur les travaux de réforme du FMI. Les conclusions du premier rapport relatif à l'utilisation prolongée des ressources de l'institution ont conduit à modifier les programmes et à rechercher une meilleure coordination avec la Banque mondiale. La Suisse a souligné avec insistance que les crédits du FMI ne devraient pas servir au financement permanent du développement.

Le deuxième rapport, consacré aux crises financières survenues en Indonésie, en Corée et au Brésil, a également relevé les points faibles de la politique suivie par le FMI. Le troisième a examiné les politiques d'ajustement fiscal que comportent les programmes financés par le FMI. Il reconnaît que le FMI évite le piège de principes rigides applicables à tous les pays et se montre capable de prendre en considération les circonstances locales; cependant, seulement la moitié des programmes ont atteint leur but.

\section{$\square$ Autres activités de la Suisse au sein du FMI}

Les Chambres fédérales ont adopté en mars (Conseil des Etats) et en juin 2003 (Conseil national) la proposition du Conseil fédéral de renouveler les accords généraux d'emprunt du FMI ${ }^{44}$. La Loi fédérale sur l'aide monétaire internationale a été adoptée en septembre 2003 par le Conseil des Etats, et en décembre

${ }^{41}$ DFF, Rapport du G10 sur les clauses d'action collective dans les contrats d'emprunts d'Etats, communiqué de presse, 8 avril 2003, <www.efd.admin.ch/d/dok/medien/medienmitteilungen $>$. Le rapport lui-même se trouve sous <www.bis.org/publ/gten08.htm>.

42 Autres solutions proposées et critique de la libéralisation du marché financier international: François Mercier, Explosive Internationale Finanzkrisen. Analysen und Lösungen im Dienst der Armutsbekämpfung, Entwicklungspolitische Impulse, nº 6/03, Bern, Brot für alle, 2003.

43 Voir le site Internet <www.imf.org/external/np/ieo $>$ et les documents qu'il contient.

44 Message concernant le renouvellement de la participation de la Suisse aux Accords généraux d'emprunt du FMI du 20 novembre 2002 (FF 2003 584) et Bulletin officiel de l'Assemblée fédérale, $<$ www.parlament.ch>. 
par le Conseil national ${ }^{45}$. Ce dernier en a toutefois limité la validité à cinq ans, créant ainsi une divergence avec le Conseil des Etats.

\subsubsection{Banque mondiale}

\section{$\square$ Activité financière}

Les opérations de crédit de la BIRD et de l'AID sont restées considérables durant l'exercice 2003, et leur volume a atteint presque celui de l'année précédente. Les remboursements se sont élevés à près du double des crédits octroyés par la BIRD, de sorte que celle-ci a enregistré un afflux net de capitaux (tableau $34)^{46}$.

Pour financer ses crédits, la Banque mondiale a emprunté un total de 18,8 milliards de dollars dans neuf monnaies différentes, principalement en dollars, en yens et en euros. Elle n'a pas présenté à part ses emprunts en francs suisses.

La $13^{\mathrm{e}}$ reconstitution du fonds de l'AID décidée l'année précédente est entrée en vigueur fin mars, après que 22 des 39 pays donateurs eurent définitivement accordé leur contribution. Le Conseil fédéral a confirmé en mai 2003 l'apport de la Suisse, qui se monte en tout à 530,7 millions de francs, les versements devant être échelonnés de 2003 à 2011. La part de la Suisse est maintenue à $2,43 \%$.

Au cours de l'exercice écoulé, la BIRD a acheté dans ses pays membres pour 1355 millions de dollars de biens et services, dont pour 54 millions (ou 3,97\%) en Suisse. Les achats analogues de l'AID ont totalisé 1498 millions de dollars, dont 12 millions $(1,1 \%)$ en Suisse.

Tableau 34: Flux de ressources de la BIRD et de l'AID, 1999-2003 (en millions de dollars, clôture au 30 juin)

\begin{tabular}{lrrrrr}
\hline & $\mathbf{1 9 9 9}$ & $\mathbf{2 0 0 0}$ & $\mathbf{2 0 0 1}$ & $\mathbf{2 0 0 2}$ & $\mathbf{2 0 0 3}$ \\
\hline BIRD & \multicolumn{7}{c}{} & & \\
\hline Engagements & 22182 & 10919 & 10481 & 11452 & 11231 \\
\hline Nombre de projets & 131 & 97 & 91 & 96 & 99 \\
\hline Versements & 18205 & 13332 & 11784 & 11256 & 11921 \\
\hline Flux de ressources nets & 8233 & 2934 & 2149 & -769 & -7956 \\
\hline Crédits non remboursés & 117228 & 120104 & 118866 & 121589 & 116240 \\
\hline AID & & & & & \\
\hline Engagements & 6967 & 4358 & 6764 & 8068 & 7282 \\
\hline Nombre de projets & 145 & 126 & 134 & 133 & 141 \\
\hline Versements & 6172 & 5177 & 5492 & 6612 & 7019 \\
\hline Flux de ressources nets & 5358 & 4257 & 4495 & 5549 & 5651 \\
\hline Crédits non remboursés & 83158 & 86643 & 86572 & 96372 & 106877 \\
\hline
\end{tabular}

Source:WB, Annual Report 2003, Washington, 2003, <www.worldbank.org/annualreport/2003>.

45 Message relatif à la Loi fédérale sur l'aide monétaire internationale du 21 mai 2003 (FF 2003 4306) et Bulletin officiel de l'Assemblée fédérale, <www.parlament.ch>.

46 WB, Annual Report 2003, Washington, 2003, <www.worldbank.org/annualreport/2003>. 


\section{$\square$ Stratégie et actions de la Banque mondiale ${ }^{47}$}

La Banque mondiale a certifié que son objectif premier reste de combattre la pauvreté. Les critères à remplir selon elle sont un Etat actif et une bonne gestion des affaires publiques (gouvernance), l'habilitation des citoyens et citoyennes (empowerment), la croissance économique, un secteur privé dynamique, un secteur financier sain, un marché mondial ouvert. Et il faut surtout que les pays en développement puissent fixer eux-mêmes leur cadence de développement (ownership).

Dans cette optique, la Banque mondiale a continué en 2003 de mettre ses priorités d'une part sur un climat favorable aux investissements. Cela implique l'encouragement des entreprises grandes et petites, la création d'emplois et l'amélioration de la productivité. La Banque s'est engagée en faveur de conditions macroéconomiques stables, d'une saine gestion des affaires publiques, d'institutions mieux organisées, d'infrastructures matérielles et financières plus solides.

Elle s'est par ailleurs employée à «investir dans les pauvres» - en aidant les plus démunis à s'informer, en leur ouvrant une participation accrue aux processus de décision, en leur donnant accès aux services. L'Etat remplit dans ce contexte une fonction essentiellement régulatrice, tandis que les services doivent être fournis par le secteur privé ou, éventuellement, par des ONG. Tels sont les principes exposés dans le rapport de la Banque mondiale sur le développement dans le monde, lequel a pourtant essuyé des critiques acerbes de la part des ONG du monde entier ${ }^{48}$. Celles-ci craignent la résurgence de projets controversés tels que les grands barrages. Elles se montrent réticentes à l'idée d'affecter des fonds d'aide à la mobilisation de capitaux privés.

La Banque mondiale a intensifié ses partenariats dans la ligne de son objectif principal et des actions qui s'y rapportent. Outre sa collaboration avec les autres institutions internationales de développement, elle a poursuivi le dialogue avec la société civile - entre autres des organisations religieuses, des ONG et des syndicats. Elle a convenu avec ceux-ci, en dépit de sérieux affrontements, des projets de recherches communes et des échanges de personnel. Elle a également organisé conjointement avec le FMI une nouvelle rencontre avec des membres de parlements nationaux, cette fois-ci à Athènes. La Suisse y était représentée par trois parlementaires, à savoir la conseillère nationale Rosmarie Zapfl et les conseillers nationaux Claude Frey et Remo Gysin.

A l'échelon national, la Banque mondiale a surtout appliqué les directives élaborées l'année précédente concernant les pays à faible revenu en difficulté (lowincome countries under stress, LICUS $)^{49}$. Elle a également étudié de nouvelles directives applicables aux pays à moyens revenus.

La Banque a poursuivi, par des mesures internes aussi bien qu'externes, les efforts entrepris pour améliorer l'efficacité du développement - par exemple en simplifiant les procédures d'octroi de crédits, au niveau de l'approvisionnement et à celui de ses propres lignes directrices politiques. Elle a aussi occupé le terrain en ce qui concerne la surveillance des objectifs du millénaire, domaine où couve d'ailleurs un conflit de méthodes entre l'ONU et la Banque mondiale.

47 Ibid.

48 WB, World Development Report 2004 : Making Services Work for Poor People, Washington, 2003, $<$ http://econ.worldbank.org/wdr/wdr2004>.

49 Concernant les LICUS, voir <www1.worldbank.org/operations/licus >. 


\section{$\square$ Coopération bilatérale entre la Suisse et la Banque mondiale}

La Suisse a poursuivi sa coopération avec la Banque mondiale: la DDC a ainsi versé 3,5 millions de francs pour un programme du World Bank Institute destiné à promouvoir l'autonomie locale dans divers domaines. Des évaluations conjointes ont lieu par ailleurs entre la DDC et l'Operation Evaluation Department $(O E D)$ de la Banque mondiale, concernant entre autres l'aide de la Banque à des pays en transition.

\subsubsection{Les sessions des institutions de Bretton Woods}

Le FMI et la Banque mondiale publient sur leurs sites Internet ordres du jour, études de base, dossiers de conférences de presse, discours et communiqués finaux de leurs réunions semestrielles ${ }^{50}$.

La session de printemps a eu lieu mi-avril à Washington. Pour la première fois, le G10 $0^{51}$ n'a pas tenu sa propre séance. La Suisse s'est opposée, conjointement avec la Belgique, les Pays-Bas et la Suède (G4 informel), à la proposition émise par le G7 de restreindre encore davantage les activités du G10.

FMI et Banque mondiale ont mis sur pied l'un et l'autre leur groupe de travail pour l'Irak. Des désaccords se sont manifestés quant aux tâches et aux compétences des IBW pour le désendettement et la reconstruction de l'Irak. Ce débat a marginalisé celui sur la politique de développement et sur les thèmes qui s'y rapportent, alors même que les deux instances avaient d'importantes études à traiter.

Le Comité du développement a discuté d'une part un plan d'action en vue d'atteindre les objectifs du millénaire ${ }^{52}$. D'autre part, il a sondé les moyens envisageables d'accorder aux pays en développement davantage de poids au sein des IBW. Les décisions à prendre ont été repoussées à l'assemblée annuelle, avec l'argument d'études complémentaires à réaliser.

Le Comité monétaire et financier international (CMFI) s'est occupé d'analyser la morosité de l'économie mondiale, et a examiné la méthode à adopter pour prévenir ou gérer des crises. Les propositions faites pour instaurer des règles internationales en matière d'insolvabilité n'ont pas reçu l'appui des Etats-Unis, de sorte qu'il n'a pas été possible de modifier les statuts du FMI comme cela aurait été nécessaire. Les efforts déployés dans ce contexte par le FMI s'en trouvent ainsi pratiquement gelés.

La session annuelle du FMI et de la Banque mondiale s'est tenue en septembre 2003 pour la première fois dans le monde arabe, plus précisément à Dubai (Emirats arabes unis). Traditionnellement, cette assemblée annuelle a lieu tous les trois ans ailleurs qu'à Washington. Les institutions de Bretton Woods ont choisi ce lieu en raison de l'importance particulière que revêtent les problèmes du Moyen-Orient et de la région méditerranéenne, Afrique du Nord comprise. Il y a d'une part la reconstruction de l'Irak, de l'Afghanistan et de la Palestine,

$50<$ www.imf.org/external/am/2003/index.htm> et <www.worldbank.org/ambc>.

51 A propos des divers groupes de pays, voir FMI, A Guide to Committees, Groups and Clubs : A Factsheet, April 2003, <www.imf.org/external/np/exr/facts/groups.htm>.

52 BM et FMI ont publié à ce sujet de volumineux documents. Voir le site du Comité du développement: <http://web.worldbank.org/WBSITE/EXTERNAL/DEVCOMMEXT/0,,menuPK:60001650 pagePK:60000303 piPK:64000842 theSitePK:277473,00.html>. 
mais aussi les difficultés économiques de toute la région - chômage, pauvreté, faible croissance, sous-développement du secteur financier et du commerce extérieur, nécessité d'impliquer les femmes dans les processus de développement. Aussi bien le FMI que la Banque mondiale ont publié de nombreux documents sur ces sujets ${ }^{53}$.

L'assemblée annuelle du Conseil des gouverneurs a été présidée pour la première fois par un Suisse : cet honneur est échu au conseiller fédéral Kaspar Villiger, qui a prononcé le discours d'ouverture ${ }^{54}$ et présidé la conférence de presse finale ${ }^{55}$.

Cette session annuelle était placée sous le signe de l'échec subi par la réunion ministérielle de l'OMC à Cancún avec la confrontation Nord-Sud, les objectifs de développement du millénaire et la lutte contre la pauvreté, ainsi que le marasme de l'économie mondiale. Autres centres d'intérêt: la reconstruction de l'Irak, les problèmes de l'Argentine, les déséquilibres du système financier international. Les ministres des Finances du G7 ont plaidé pour une flexibilité accrue des cours du change - sur fond de critique des Etats-Unis à l'égard de la Chine, dont la monnaie est liée au dollar. On reproche aux autorités chinoises de maintenir ainsi leur monnaie à un niveau artificiellement bas. Le G7 a par ailleurs exhorté tous les pays à ouvrir l'accès à leurs informations bancaires pour combattre le blanchiment d'argent et la fraude fiscale, une revendication qui était dirigée entre autres contre la Suisse ${ }^{56}$.

Le Comité monétaire et financier international a discuté comme de coutume la situation de l'économie mondiale et examiné les instruments du FMI pour la surveillance, pour la prévention et la gestion de crises, pour la stabilisation du marché international des capitaux. Le conseiller fédéral Kaspar Villiger a critiqué notamment la fait que le FMI immobilise trop d'argent dans quelques grands pays, au détriment d'autres régions ${ }^{57}$.

Le Comité $d u$ développement a traité principalement trois sujets ${ }^{58}$. FMI et Banque mondiale ont premièrement exhorté les pays riches à aider ceux qui pratiquent une bonne politique macroéconomique en leur apportant un soutien financier approprié dans le cadre des DSRP, de manière à atteindre les objectifs du millénaire. M. Wolfensohn, président de la Banque mondiale, estime à 16 milliards de dollars par année jusqu'en 2016 le supplément d'aide publique nécessaire pour financer le développement.

En deuxième lieu, le Comité du développement s'est penché sur la question de savoir comment renforcer la présence et la participation des pays en développement au sein de la Banque et du FMI. La répartition des droits de vote reste

53 Concernant les activités de la BM et du FMI au Moyen-Orient et en Afrique du Nord, voir <www.worldbank.org/mna $>$ et <www.imf.org/external/pubs/ft/med/2003/eng/creane/index.htm > . Voir aussi : IMF, Finance and Development, vol. 40, $\mathrm{n}^{\circ}$ 1, March 2003, <www.imf.org/fandd $>$.

$54<$ www.efd.admin.ch/f/dok/referate/2003/09/iwf.htm>.

$55<$ www.imf.org/external/am/2003/speeches/pr62e.pdf>.

56 Statement of G7 Finance Ministers and Central Bank Governors, Dubai, 20 September 2003, $<$ www.g7.utoronto.ca/finance/fm030920.htm>.

57 Communiqué du CMFI, 21 septembre 2003, <www.imf.org/external/np/cm/2003/092103a.htm>. Déclaration de Kaspar Villiger au CMFI : <www.imf.org/external/am/2003/imfc/state/che.htm>.

58 Communiqué du Comité du développement, 22 septembre 2003, <www.imf.org/external/np/cm/2003/ 092203f.htm>. Déclaration de la Suisse (Oscar Knapp, Seco) au Comité du développement, 22 septembre 2003, <http://web.worldbank.org/WBSITE/EXTERNAL/DEVCOMMEXT/0,,menuPK: 60001658 pagePK:64046478 piPK:64046507 theSitePK:277473,00.html>. 
inchangée; seules des mesures d'appui administratif et financier ont été décidées. La Suisse entend s'y associer, mais serait aussi disposée à envisager une réévaluation du droit de vote de base. Troisièmement, le Comité du développement a discuté en détail la situation telle qu'elle se présentait après l'échec de la Conférence ministérielle de l'OMC.

Il n'y a eu aucune manifestation à Dubaï, mais en revanche de nombreuses rencontres entre des ONG et les IBW ${ }^{59}$.

Avant la session annuelle s'est tenue, également à Dubaï, une réunion du groupe de vote dirigé par la Suisse. Ce groupe a débattu des expériences faites par les pays qui le composent en matière de stratégies de réduction de la pauvreté (SRP). Le Tadjikistan, l'Azerbaïdjan et le Kirghizistan ont déjà leur DSRP, tandis que la Serbie et le Monténégro sont actuellement en train d'élaborer leur document stratégique. La DDC y a présenté son étude sur l'application du DSRP dans quatre pays prioritaires de la Suisse (dont le Kirghizistan) ${ }^{60}$.

\section{SOURCES}

Institutions de Bretton Woods

Communiqué du Comité du développement, <www.imf.org/external/np/cm/2003/092203f.htm>.

IMF/WB, Guidelines for Public Debt Management, <www.imf.org/external/np/mae/pdebt/2002/eng/ 102402.htm>.

IMF/WB, Heavily Indebted Poor Countries (HIPC) Initiative: Status of Implementation, Washington, 12 September 2003, <www.imf.org/external/np/hipc/2003/status/091203.htm>.

IMF/WB, Report on Joint Bank-Fund Workshop on Debt Sustainability in Low-Income Countries, Washington D.C., 11-12 September 2003, <www.imf.org/external/np/pdr/sustain/2003/100903.htm>.

IMF/WB, Development Committee, Recent Trends in Financial Flows to Developing Countries, Washington, 27 August 2003, <www.worldbank.org/ambc>.

Sites de la session annuelle: <www.imf.org/external/am/2003/index.htm > et <www.worldbank.org/ambc >

Fonds monétaire international <www.imf.org $>$

A Guide to Committees, Groups and Clubs: A Factsheet, April 2003, <www.imf.org/external/np/exr/ facts/groups.htm>.

Annual Report 2003, Washington, 2003, <www.imf.org/external/pubs/ft/ar/2003/eng/index.htm>.

Communiqué du développement, 22 septembre 2003, <www.imf.org/external/np/cm/2003/092203f.htm>.

Executive Director for Azerbaijan, Kyrgyz Republic, Poland, Serbia and Montenegro, Switzerland, Tajikistan, Turkmenistan, Uzbekistan, Activity Report 2003,Washington, September 2003, p. 26.

Global Financial Stability Report, divers numéros, Washington, <www.imf.org/external/pubs/ft/gfsr/ about.htm>.

Switzerland: 2003 Article IV Consultation: Staff Report..., Country Report, nº 03/148, 2 June 2003, $<w w w . i m f . o r g /$ external/pubs/cat/longres.cfm?sk=16579.0>.

Switzerland-2003 Article IV Consultation: Concluding Statement, Bern, 24 February 2003, <www. imf.org/external/np/ms/2003/022403.htm>.

World Economic Outlook: Growth and Institutions, April 2003, <www.imf.org/external/pubs/ft/weo/ 2003/01>.

World Economic Outlook: Public Debt in Emerging Markets, September 2003, <www.imf.org/external/ pubs/ft/weo/2003/02>.

59 Voir Transcript of a Townhall Meeting with CSOs, 21 September $2003:<w w w . i m f . o r g / e x t e r n a l / n p /$ tr/2003/tr092103a.htm>.

60 Voir plus haut, dans la partie 7.4.1, le point «DSRP - un principe qui s'impose peu à peu». 


\section{Banque mondiale <www.worlbank.org $>$}

Annual Report 2003, Washington, 2003, <www.worldbank.org/annualreport/2003>.

Executive Director for Azerbaijan, Kyrgyz Republic, Poland, Serbia and Montenegro, Switzerland, Tajikistan, Turkmenistan, Uzbekistan, Report to the Governors for the Annual Meetings, September 2003, Washington.

Global Development Finance: Striving for Stability in Development Finance, vol.1: Analysis and Statistical Appendix, Washington, 2003, <www.worldbank.org/prospects/gdf2003/tocvol1.htm>.

Global Economic Prospects : Realizing the Developing Promise of the Doha Agenda, Washington, 2003, <www.worldbank.org/prospects/gep2004>.

OED, The Heavily Indebted Poor Countries (HIPC) Initiative: An OED Review, Report, $\mathrm{n}^{\circ} 25160$, Washington, 20 February 2003, <www.worldbank.org/oed>.

World Development Report 2004 : Making Services Work for Poor People, Washington, 2003, <http:// econ.worldbank.org/wdr/wdr2004>.

Banque des règlements internationaux <www.bis.org >

Diverses publications telles que rapport annuel, rapports trimestriels, etc.

OCDE <www.oecd.org>

Club de Paris <www.clubdeparis.org $>$

ONU

UNCTAD, Trade and Development Report 2003 : Capital Accumulation, Growth and Structural Change, UN, Geneva and New York, 2003, <www.unctad.org >.

Site Internet de Financing for Development (FfD) : <www.un.org/esa/ffd $>$.

\section{Sources officielles suisses}

Banque nationale suisse, La balance suisse des paiements 2002, Zurich, août 2003, <www.snb.ch/f/publikationen/publi.html>.

Commission de gestion du Conseil des Etats, La Suisse comme membre des institutions de Bretton Woods, Berne, 14 octobre 2003, <www.parlament.ch/f/ed-pa-gpk-s-brettonwoods-d.pdf>.

Conseil fédéral, Rapport sur la politique économique extérieure 2002, 15 janvier 2003, <www.seco. admin.ch/publikationen>.

DDC, SDC's Bilateral Engagement in the Poverty Reduction Strategy Paper (PRSP) Process. Evaluation, 2003/1, <www.deza.admin.ch>.

Déclaration de Kaspar Villiger au CMFI : <www.imf.org/external/am/2003/imfc/state/che.htm>.

Déclaration de la Suisse (Oscar Knapp, seco) au Comité du développement : <http://web.worldbank.org/ WBSITE/EXTERNAL/DEVCOMMEXT/0,,menuPK:60001658 pagePK:64046478 piPK:64046507 $\sim$ theSitePK:277473,00.html>.

DFF, Discours d'ouverture de M. Kaspar Villiger, président des Conseils des gouverneurs et gouverneur de la Banque et du Fonds pour la Suisse, en séance plénière, <www.efd.admin.ch/f/dok/referate/ 2003/09/iwf.htm>.

DFF, Le FMI ne constate aucun déséquilibre fondamental dans l'économie suisse, communiqué de presse, 24 février 2003, <www.efd.admin.ch/f/dok/medien/medienmitteilungen/2003/02/iwf.htm>.

DFF, Newsletter concernant le FMI, <www.efv.admin.ch/f/internat/iwf_int/index.htm >.

DFF, Rapport du G10 sur les clauses d'action collective dans les contrats d'emprunts d'Etats, communiqué de presse, 8 avril 2003, <www.efd.admin.ch/d/dok/medien/medienmitteilungen>.

Message concernant le renouvellement de la participation de la Suisse aux Accords généraux d'emprunt du FMI du 20 novembre 2002 (FF 2003 584).

Message relatif à la Loi fédérale sur l'aide monétaire internationale du 21 mai 2003 (FF 2003 4306).

\section{Autres}

Baker, Dean, Morrissey, Monique, When Rivers Flow Upstream: International Capital Movements in the Era of Globalization, Briefing Paper, Centre for Economic and Policy Research (CEPR), Washington, 22 March 2003, <www.cepr.net/capital_flows.htm>.

Greenhill, Romilly, Sisti, Elena, Real Progress Report on HIPC, London, NEF/Jubilee, September 2003, $<w w w . j u b i l e e r e s e a r c h . o r g>$.

HM Treasury/Department for International Development (UK), International Finance Facility, London, January 2003, <www.hm-treasury.gov.uk/documents/international_issues/int_gnd_intfinance.cfm>.

Hochhuth, Stefan, The Impact of Debt Relief: Does the HIPC Initiative Help in Meeting the Millennium Development Goals?, Discussion Paper, London, 14 October 2003 (manuscrit non publié, rédigé en collaboration avec New Economic Foundation/Jubilee2000).

IIF, Capital Flows to Emerging Markets Economies, divers numéros, <www.iif.com>. 
Mercier, François, Explosive Internationale Finanzkrisen. Analysen und Lösungen im Dienst der Armutsbekämpfung, Entwicklungspolitische Impulse, nº 6/03, Bern, Brot für alle, 2003.

Pettifor, Ann (ed.), Real World Economic Outlook. The Legacy of Globalization: Debt and Inflation, Jubilee Research at the New Economics Foundation, Hampshire and New York, Palgrave Macmillan, 2003.

Rome Declaration on Harmonization: High Level Forum on Harmonization, Rome, 24-25 February 2003, <www1.worldbank.org/harmonization/romehlf>.

Statement of G7 Finance Ministers and Central Bank Governors, Dubai, 20 September 2003, <www.g7.utoronto.ca/finance/fm030920.htm>. 